\title{
Implementation of effective Methods of Modeling Tools in technical Education
}

\author{
Josef Sedivy \\ University of Hradec Kralove, Faculty of Science, Department of Informatics, Rokitanskeho 62, 500 \\ 03 Hradec Kralove, Czech Republic \\ josef.sedivy@uhk.cz
}

Keywords: Design, Interdisciplinary relations, Project oriented education, Simulations, Engineering, Virtual prototype.

\begin{abstract}
Development didactics engineering subjects based on the results and conclusions research studies focused on the use of didactic potential of the technology deployed in industrial environments. The possibility of good careers in the field of construction and technical preparation of engineering companies is the goal of education of students, future graduates of technical and information technology graduates secondary schools, colleges as well as universities.
\end{abstract}

\section{Introduction}

The level of preparation of the technical staff, aimed at achieving capability independent use of knowledge in the field while applying skills in using progressive tools, it is often the key to getting job and subsequent professional development [1]. The use of effective tools and methods in teaching is also a measure of the quality of individual schools and entire systems engineering didactics subjects. The research, presented in text, Based on the experiment, the deployment of tools for computer-aided design - CAD and Simulation - CAE project oriented teaching engineering subjects. It draws on the theoretical bases of engineering subjects taught [2] and the potential deployment of applied and general information technology in education [3]. Research practices in the school environment, methods, structure projects and tests are based on a research survey conducted in industrial environments, focusing on efficient use of engineering tools for solving real-world projects. The conclusions of the present research in the school environment can be used to optimize the didactic system of professional planning and engineering subjects teaching at secondary schools, colleges of engineering, information- engineering and related technical oriented focus.

\section{Modeling and simulation in project education}

The use didactic potential of information technology based on current trends in contemporary education [4].

Computer applications for design and simulation are the primary tools used in practice for the stage design and the technical preparation of the product. The product may be an individual component alone or the whole assembly of components of engineering equipment, or any other type of construction, e.g. tool. Comprehensive use of these technologies beyond the realm of engineering. Intensive use can be recorded, for example in construction and potential use is in the field of natural sciences or medicine. [5] Digital 2D, but also 3D models of real objects created tools for design and verification tools for simulation, represent a virtual prototypes. To create models use tools for design - CAD (Computer Aided Design). In the current industrial practice to use CAD application for 2D curved, or 3D surface models and solid bodies. Trend, based on the development of applications and the performance of commercially available hardware tends to use of 3D systems. Said starting demonstrate the need for the deployment of 3D CAD tools at all levels of technical and information-technically oriented schools.

Calculations, and functional verification of the characteristics of the proposed design is done through simulation tools - CAE (Computer Aided Engineering). Calculations carried out on digital 
models are based on algorithms Finite Element Method - FEM (Finite Element Method). CAE simulations can be carried out in compliance with certain principles and rules of any deep knowledge of the theory of FEM, which is especially important in secondary schools. [6] These modules can be made available separately, but it is advantageous to have comprehensive applications in which a wide range of integrated tools needed for most stages of development and production preparation product. To zoom in an environment of industrial experience shows the benefits of project-based learning, based on the solution of difficult variably complex problems presented in detail in [7]. According to the current curriculum objectives and time allocation for a particular thematic unit can be solved e.g. the following types of projects:

- Detailed design of one component, including the creation drawings and manufacturing process.

- Conceptual design for functional assemblies assessment of individual components or whole.

- Detailed design reports any size with detailing the components, including strength calculations and drawings documentation.

In all cases they should be designing projects and entered with the requirement of representation in all actions and activities based on previous theoretical foundations of their field of study. An example of a project that meets specified conditions, is shown in Fig. 1. At the consolidation of interdisciplinary relations and reinforcing the structures of knowledge [8] due to the deployment of those teaching methods and the use of sophisticated design tools aimed also presented research. Especially in terms of assessing the benefits increase and consolidate knowledge engineering issues in connection with skills using CAD and CAE tools in solving problems. This is especially the knowledge acquired in the basic courses:

- Mechanical Engineering.

- Engineering technology.

- Development of technical documentation.

- Technical mechanics, flexibility, strength.

- Technology specialization field.

- Working with CAD

- Mathematical and physical basis, the relevant requirements of solved problems.

Knowledge tests used in the research survey includes questions on topics mentioned in context with design and technological processes deployment CAx applications. Key to the qualitative assessment of pupils' final projects are also created with regard to technical expertise required structure of knowledge.

\section{Aim of the research and hypothesis formulation}

The aim of the research is to increase the effectiveness of teaching technical staff using CAx applications in teaching of technical, engineering-oriented subjects at secondary schools and colleges. The main research problem poses the question:

"Increases classroom project, supported by the deployment tools for design and simulation knowledge and skills of graduates?" Related Derivatives particular research questions are formulated as follows:

- Strengthens project teaching, supported by tools for design and simulation, inter-industry relations?

- Increases work with computer tools design and simulation of motivation and active attitude of pupils?

In the long research study, undertaken in the Secondary School and the College of Information-engineering focus is applied a mixed approach of combining qualitative and quantitative methods. Along with the evaluation tests knowledge of students was conducted a qualitative analysis 
of a number of student projects quantification of selected parameters, their evaluation and statistical evaluation.

In the first phase of the research was carried out piloting specialized in teaching engineering subjects, basic work tools for design - CAD education and information technology.

In the pre-research experiment was on a small sample of student piloted test the level of knowledge and skills. Students were again divided into two parallel groups. Students in the experimental group was in line with the curriculum of the course explain the issue of the theme with the support of 3D CAD tools. Active involvement of pupils at this stage was minimal, it was only the interpretation of a certain degree of interactivity. Pupils in the control group attended classes "classic" method, with the support of printed materials and interpreting without using a computer.

Based on the piloting and pre-research experiment in the first phase formulated hypothesis, the acceptance of which has been verified by subsequent research survey:

$\mathrm{H}_{1}$ : Use computer applications for design and simulation in teaching engineering subjects leads to increased knowledge and skills of graduates to solve engineering design problems.

Adoption hypothesis $\mathrm{H}_{1}$ depends on the assessment of two factors - knowledge and skills. The relationship of both parameters is confirmed from the beginning, so they were subsequently formulated two hypotheses:

$\mathrm{H}_{1}$ : Use computer applications for design and simulation project teaching engineering subjects leads to an increase in knowledge engineering graduates.

$\mathrm{H}_{2}$ : Use computer applications for design and simulation project teaching engineering subjects leads to an increase in the skills of graduates to solve design engineering problems.

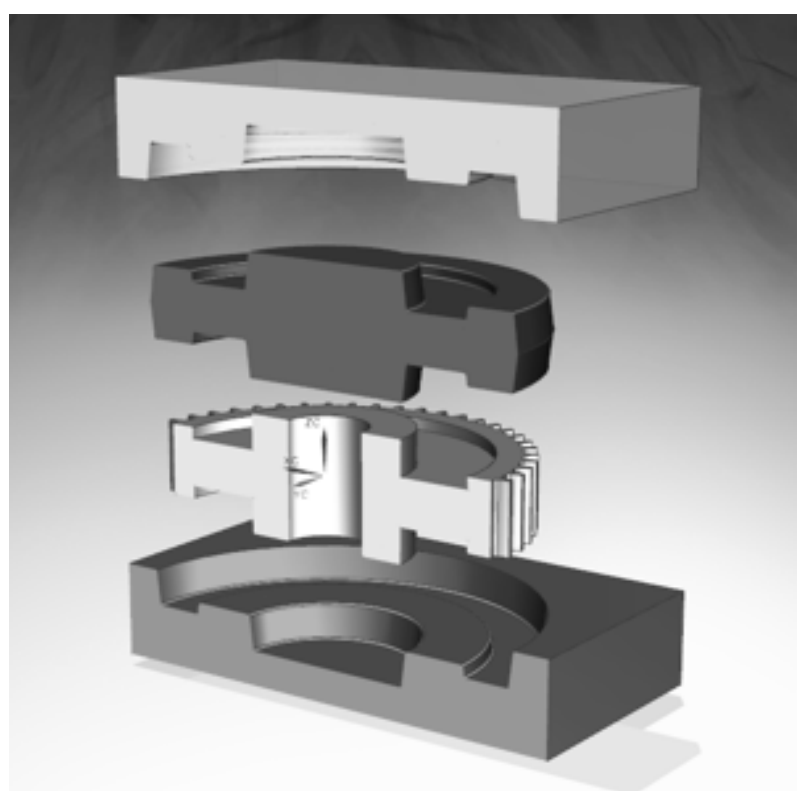

Fig.1 CAD assembly of basic components of the technological process of production of a gear.

\section{Research data}

When planning the research was carried out after the balance sheet in connection with the anticipated conditions and organizational matters after securing the long-term context chosen method of two parallel groups. Was divided into groups according to the criteria that was not associated with the problem. Usually, groups were formed under their division for teaching a foreign language or information technology within the classroom. The division into groups in different classes have been combined to equalize the number of both types of groups (experimental control). The important thing was minimal influence organizational factors i.e. the teaching should be in all groups always uniformly continuous. The resulting numbers of pupils in divide groups are shown in Table 1 below. While the number of samples can be performed with acceptable reliability evaluation to generate 
conclusions. One of the necessary conditions are the same initial knowledge and skills in both groups. This requirement was checked pretest.

Table 1 The number of samples in groups

\begin{tabular}{|l|l|}
\hline Groups & Count \\
\hline Experimental group & 52 \\
\hline Control group & 56 \\
\hline Total count & 108 \\
\hline
\end{tabular}

Unlike the output of knowledge in both groups after learning cycle was controlled posttest. Initial assay contained 54 questions and examples. In the phase of preliminary research and piloting was reduced to 45 items, 30 of which were focused on general knowledge of engineering and 15 questions respectively. Examples was focused on the verification of skills. Questions on knowledge has been compiled according to experience from practice and covered a cross-sectional core areas with which students in professional practice and recent graduates in practice most experienced. To develop and pretest posttest knowledge was fixed period of 45 minutes - one lesson. Questions with one correct answer, focusing on the facts have been rated 0 or 1 point. Part of questions to assess the skills were simple tasks solved through CAx tools. To develop tests were scheduled time 90 minutes - two hours. Examples and creative answers were rated 0.1 or 2 points. In total, the test can get 0-60 points, including the section on 30 points knowledge and skills also 30 points. The survey was repeated in three cycles, while the emphasis on ensuring equal conditions of teaching and testing of both groups. Condition for inclusion in the sample group was passing the pretest and posttest. Pupils who attended only one test, were excluded from the statistical evaluation. Their projects, however, were used for qualitative research skills to work with CAD and analysis of applied design procedures and related processes.

\section{Research and evaluation results}

Pretest results were tabulated and evaluated in MS Excel using the option "Data Analysis". The graphs were generated through the software tool Statistica. Interim results on the ground were still controlled by statistical functions Casio graphical calculator. Calculation methods based on the general procedures of processing statistical data in relation to the specifics of educational research. Evaluated knowledge pretest results are shown in Table 2. The objective was to compare the initial parameters of control and experimental groups with each other to confirm the assumption of an independent choice. In this phase of testing was considered approximately equal knowledge of engineering issues in accordance with the above rules.

For the purpose of monitoring and statistical evaluation of the chosen method was formulated by a working null hypothesis:

$\mathrm{H}_{0}$ : Knowledge listeners in control and the experimental group are the same compared to the alternative hypothesis:

$\mathrm{H}_{\mathrm{A}}$ : Knowledge listeners in control and the experimental group are different.

To assess the acceptance of the null hypothesis $\mathrm{H}_{0}$ against alternative hypothesis $\mathrm{HA}$ was chosen two-sided t-test with a significance level of 0,05 and 0,01 . The same procedure was also used in the evaluation of the results of the posttest. At this stage, the adoption of the alternative hypothesis $\mathrm{H}_{\mathrm{A}}$ while a higher success rate of the experimental group mean direct acceptance hypothesis $\mathrm{H}_{1}$. Graphical representation of evaluation pretest and posttest in both types of groups in the graph on fig. 2. 


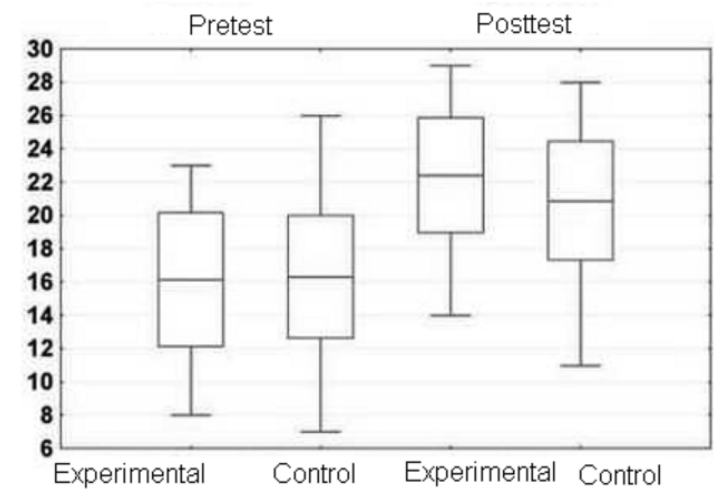

Fig. 2 Graphical representation of data evaluation, testing knowledge of engineering issues

The t-statistic is pretest results in the field of adoption of the hypothesis $\mathrm{H}_{0}$ for a two-sided test at a significance level of 0,05 . You can accept the default hypothesis $\mathrm{H}_{0}$ and knowledge of both groups consider the same.

Posttest evaluation directly leads to the acceptance or rejection of the hypothesis $\mathrm{H}_{1}$. Results were evaluated by one-sided t-test at a significance level of 0,05 and 0,01 .

The t-statistic is 2,088 critical field admission at a significance level of $0,05 . \mathrm{H}_{0}$ hypothesis at this significance level we reject, but it can be taken on a significance level of 0,01 . Based on the statistical evaluation introduced by not clearly accept the hypothesis $\mathrm{H}_{1}$ increasing engineering knowledge.

For pretest skills and relationships were formulated by a working null hypothesis:

- $\mathrm{H}_{0}$ : Skills solving engineering tasks are for students in the experimental and control group of the same compare to the alternative hypothesis:

- $\mathrm{H}_{\mathrm{A}}$ : Skills solving engineering tasks are for students in the experimental and control groups are different.

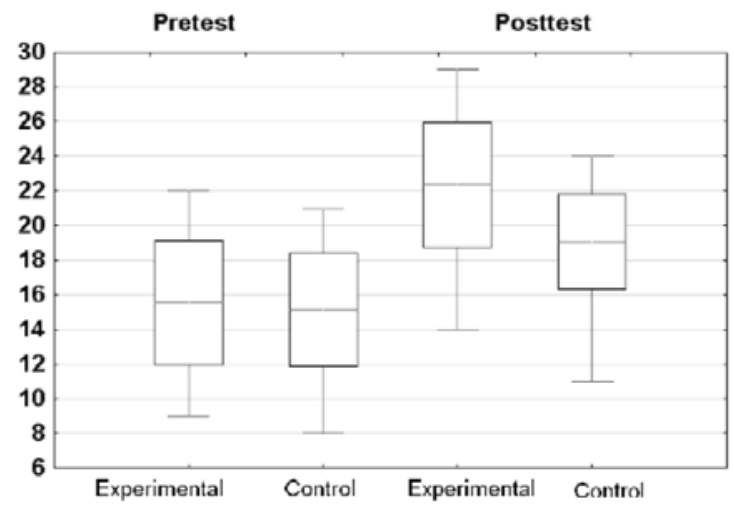

Fig. 3 Graphical representation of data evaluation, skills testing solutions engineering tasks

The t-statistic evaluated results pretest located reliably in the field of acceptance of the null hypothesis at a significance level of 0,05. Default skills groups at this stage be considered the same. Based on processed and evaluated the results of the posttest will be decided on the acceptance or rejection of the hypothesis $\mathrm{H}_{2}$. The results were analyzed by t-test at a significance level of 0,05 and 0,01 .

The t-statistic is 4,81 in the art reject the hypothesis $\mathrm{H}_{0}$ at a significance level of 0,05 and 0,01 . Results of the experimental groups is based on a comparison of mean values better than the results in the control group. Based on the results of the investigation that method reject the hypothesis $\mathrm{H}_{0}$ of conformity mean values in favor of the hypothesis $\mathrm{H}_{\mathrm{A}}$. Since further assessed conditions may accept the hypothesis $\mathrm{H}_{2}$ to increase the skills of solving engineering tasks. 


\section{Conclusion}

The conclusions of the research conducted and presented methods under these conditions allow to accept the hypothesis of increased skills to solve design engineering problems. This assertion can be supported and experienced graduates in practice and comparing the results obtained work on school projects with requirements for outputs work of designers. Conversely, acceptance of the hypothesis enhance knowledge of engineering issues at this stage is still debatable. The measurement results according to the test corroborate the claim. Thorough analysis of project outcomes, it was found that in the context of longer-term labor listeners can the facts in the particular context practically applied. Due to the methodology of the research, this factor supports the adoption of assumptions about knowledge industry. Educators can, on the basis of research results apply their own creativity to prepare the sub-themes of teaching technical subjects, or large-scale long-term individual and team projects.

\section{Acknowledgment}

This article was created under the project called "Research team in didactic" done at UHK, faculty of Science, Hradec Kralove in 2015.

\section{References}

[1] W. Qiu, Study on the Normalization for Internet Computing and Modeling of Conceptual Model XML-Based, In: Advanced Materials Research, Vol. 121-122, 2010.

[2] J. W. Yu, New Approach for Software Modeling and Transformation, In: Applied Mechanics and Materials, Vol. 546-547, 2012.

[3] J. W. Yu, New Approach for Software Modeling and Transformation, In: Applied Mechanics and Materials, Vol. 546-547, 2012.

[4] H. L. Geng, M. Tian: Integration of Modern Information Technologies and Mathematics Teaching, In: Advanced Materials Research, Volume: Advanced Research on Automation, Communication, Architectonics and Materials, 2011.

[5] P. Vobornik, Mini-Language for Effective Definition of the Color Gradients, In: Advanced Materials Research, Vols. 1030-1032, pp. 1882-1885, 2014.

[6] Hubalovsky, S., Modeling and Simulation of Real Process - Passing through the Labyrinth as a Method of Development of Algorithm Thinking and Programming Skills. In: International journal of mathematics and computers in simulation. p. 125-133, 2013.

[7] J. C. Geng, S. X. Niu, Z. X. Li, Using Enterprise Architecture to Direct IT Governance, Advanced Materials Research, Vols. 971-973, pp. 2452-2457, 2014.

[8] M. Maněnová, V. Tauchmanová, Analysis of Communication Tools of the Learning Management Systems of Moodle and WebCt. In Education and educational technology (EDU'11). Athens: World scientific and engineering academy and society, s. 82-86, 2011. 